\title{
Mild Hydrothermal Treatment to Prepare Highly Dispersed Multi-walled Carbon Nanotubes
}

\author{
Li Zhang ${ }^{\text {a }}$, Yoshio Hashimoto ${ }^{\text {a }}$, Toshinori Taishi ${ }^{\text {a }}$, Qing-Qing Ni ${ }^{\text {b }}$ \\ ${ }^{a}$ Faculty of Engineering, Shinshu University, Japan \\ ${ }^{\mathrm{b}}$ Dept. of Functional Machinery \& Mechanics, Shinshu University, Japan
}

\begin{abstract}
Multi-walled carbon nanotubes (MWCNTs) with improved dispersion property have been prepared by a mild and fast hydrothermal treatment. The hydrothermal process avoids using harsh oxidants and organic solvents, which is environmental friendly and greatly decreases the damage to intrinsic structure of MWCNTs. The modified MWCNTs were highly soluble in polar solvents such as water, ethanol and dimethylformamide. Morphological observation by TEM indicated that the diameter and inherent structure were well reserved in modified MWCNTs. X-ray photoelectron spectroscopy and Raman spectroscopy were used to quantify functional groups created on the MWCNT surface, and to determine rational parameters of hydrothermal process.
\end{abstract}

Keyword: Multi-walled carbon nanotubes; Hydrothermal treatment; Improved dispersion property

\footnotetext{
${ }^{*}$ Corresponding author at: Dept. of Functional Machinery \& Mechanics, Shinshu University, 3-15-1 Tokida, Ueda 386-8567, Japan. Tel/Fax: +81268215438

E-mail address: niqq@shinshu-u.ac.jp (Qing-Qing Ni)
} 


\section{Introduction}

Multi-walled carbon nanotubes (MWCNTs) with their unique structure and properties are well known for the wide range of potential applications, from composite materials to nanodevices [1-6]. Unfortunately, because of the strong intrinsic van der Waals attractions, MWCNTs have limited solubility in most organic and aqueous solvents. The poor chemical compatibility greatly hinders their solution phase manipulation and processability. Extensive researches have thus focused on the MWCNT modification to provide homogeneous dispersion of MWCNTs in various solutions [7-13], mainly in term of severe oxidation treatments $\left(\mathrm{H}_{2} \mathrm{SO}_{4}\right.$ [10], $\mathrm{HNO}_{3}$ $[11,12]$ or $\left.\mathrm{KMnO}_{4}[13]\right)$ that allow the introduction of carboxyl and hydroxyl groups to MWCNT surface. In general, these approaches unavoidably break carbon nanotubes into short fragments and even change their inherent morphology owing to oxidative conditions. Moreover, these treatments entail environmental problems, such as the production of acid effluent.

Therefore, a facile, eco-friendly and less destructive modification route is still of great interest. In our previous study [14], functional groups have been successfully introduced to the surface of single-walled carbon nanotubes via the oxidation process using potassium persulfate (KPS). However, in that case, the process was still timeconsuming, and modification parameters were not discussed. Herein, we conduct the MWCNT modification under hydrothermal condition to accelerate the reaction, and the effective surface modification could be achieved within $0.5 \mathrm{~h}$. Furthermore, the effect of reaction parameters on MWCNT modification is systematically discussed. The modification process could be a practical strategy to prepare highly dispersed MWCNTs, and paves the way for the wide potential application of MWCNTs.

\section{Experimental}

MWCNTs (purity $>90 \%$ ) with diameters of 20-40 nm were used as received. All other reagents were of analytical grade and were used without further purification. We conducted a total of eleven experiments. The detailed reaction parameters and corresponding analyses are listed in Table 1 . The procedures of all the experiments were similar, so we took the No.8 experiment as an example to present the detailed procedure. 
We mixed pristine MWCNTs $(30 \mathrm{mg}$ ) with an aqueous solution $(50 \mathrm{ml})$ of KPS $(0.05 \mathrm{M})$ and $\mathrm{KOH}(0.15 \mathrm{M})$, and measured the $\mathrm{pH}$ of the solution using a $\mathrm{pH}$ meter (Model TPX-999, ToKo Chemical Laboratories Co., Ltd.). After 10 min of ultrasonic mixing, the solution was transferred into a stainless steel reaction autoclave $(50 \mathrm{ml})$ with polytetrafluoroethylene liner. The autoclave was sealed and heated to $150{ }^{\circ} \mathrm{C}$ at a rate of $10{ }^{\circ} \mathrm{C} / \mathrm{min}$ and then maintained at $150{ }^{\circ} \mathrm{C}$ for $0.5 \mathrm{~h}$. After being cooled to room temperature, we diluted and centrifuged the product solution. The supernatant solution was collected and filtered through a hydrophilic polytetrafluoroethylene membrance ( $47 \mathrm{~mm} / 0.2 \mu \mathrm{m}$, Milllipore Corp., USA), and the product was rinsed with deionized water and ethanol for several times to remove the ions possibly remaining in the products. Finally, the product was dried overnight in vacuum at $30{ }^{\circ} \mathrm{C}$.

We performed XPS measurement on a Kratos Axis Ultra DLD X-ray photoelectron spectrometer with a standard $\mathrm{MgK} \alpha(1256.6 \mathrm{eV}) \mathrm{X}$-ray source operated at $10 \mathrm{~mA}$ and $15 \mathrm{kV}$, and referred all binding energies to $\mathrm{Au} 4 \mathrm{f}_{7 / 2}$ at $83.8 \mathrm{eV}$. The XPSPeak 4.1 software with Gaussian-Lorentzian mix function and Shirley background subtraction was used to analyze C 1s spectra quantitatively. Peak positions of the samples were reproducible using a fixed Gaussian to Lorentz ratio of 80:20 and fixed FWHMs. Raman spectroscopy measurement was carried out with a HoloLab series 5000 Raman spectroscopy (Kaiser optical systems, Inc.) furnished with $532 \mathrm{~nm}$ laser excitation ( 5 specimens of each material were tested for reproducibility). Zeta potential for the modified MWCNTs was measured at a concentration of $0.01 \mathrm{wt} . \%$ using a Zetaplus analyzer (NanoZS, Malvern company, Britain) by electrophoresis light scattering method. We imaged the samples with a field emission scanning electron microscopy (FESEM, Hitachi S-5000, $20 \mathrm{kV}$ ) and transmission electron microscopy (TEM, JEM-1010, 200kV). Thermogravimetrical analysis (TGA) was performed on a Rigaku Thermo Plus TG8120 apparatus (Rigaku Denki, Japan) under air/nitrogen atmosphere at $10^{\circ} \mathrm{C} / \mathrm{min}$ in a temperature rang of $30-800{ }^{\circ} \mathrm{C}$.

\section{Results and discussion}

\subsection{Reaction parameters of MWCNT modification}

A series of modified MWCNTs were prepared by hydrothermal treatment conducted under different parameters (Table 1: Nos. 1-4, Nos. 5-7 and Nos. 8-11 
with different KPS concentration, $\mathrm{KOH}$ concentration and reaction time, respectively). We investigated the effect of KPS on CNT modification by controlling its concentrations at $0.01,0.05,0.1$ and $0.2 \mathrm{M}$ with the $\mathrm{KPS} / \mathrm{KOH}$ ratio at $1: 3$ (Nos.1-4 in Table 1; the reason for $\mathrm{KPS} / \mathrm{KOH}$ ratio at 1:3 will be discussed later in this paper). XPS analysis provides direct information of elemental composition and functional groups on MWCNT surface resulting from the hydrothermal oxidation at different KPS concentration. Figure 1a shows the XPS survey scans of the modified MWCNTs in comparison to the pristine ones. All modified MWCNT spectra showed a strong C 1s peak at about $284 \mathrm{eV}$, and all the element in the samples were carbon (C 1s), oxygen $(\mathrm{O} 1 \mathrm{~s})$ and potassium $(\mathrm{K} 2 \mathrm{p}, \mathrm{K} 2 \mathrm{~s})$. In addition, the percentage of the oxygen atoms increased as a function of KPS concentration, corresponding to the distribution of oxygen atom in survey spectra. Figure $1 \mathrm{~b}$ presents the high-resolution $\mathrm{C} 1 \mathrm{~s}$ spectra of pristine MWCNTs and the modified MWCNTs obtained by hydrothermal treatment in different KPS concentration. On the basis of the literature reports [7,1518], the overall $\mathrm{C} 1 \mathrm{~s}$ peak was fitted by a superposition of five peaks (the $\mathrm{K} 2 \mathrm{p}$ peaks at $293.2 \mathrm{eV}$ and $295.9 \mathrm{eV}$ were taken into account during fitting, because it affected the area of $\mathrm{C} 1 \mathrm{~s}$ spectrum). The main peak at $284.3 \mathrm{eV}$ could be assigned to $\mathrm{sp}^{2}-\mathrm{C}-$ $\mathrm{C}-$, and the other four peaks were attributed to $\mathrm{sp}^{3}-\mathrm{C}-\mathrm{C}-(284.7 \mathrm{eV}),-\mathrm{C}-\mathrm{O}-(286.2$ $\mathrm{eV}),-\mathrm{C}=\mathrm{O}(287.5 \mathrm{eV})$ and $-\mathrm{COO}-(289.2 \mathrm{eV})$. To assess the amount of different functional groups created on the MWCNT surface quantitatively, the relative percentage was calculated based on the ratios of peak areas (Figure $1 \mathrm{~b}$ or Table 1). The treatment of KPS concentration at $0.01 \mathrm{M}$ (No.1 in Figure $1 \mathrm{~b}$ ) created a relatively small amount of functional groups $(-\mathrm{C}-\mathrm{O}-, 6.4 \% ;-\mathrm{C}=\mathrm{O}, 2.0 \% ;-\mathrm{COO}-, 7.9 \%)$. Treating the MWCNT at the KPS concentration of $0.05 \mathrm{M}$ increased the amount of functional groups reaching its approximate maximum value $(-\mathrm{C}-\mathrm{O}-, 7.6 \% ;-\mathrm{C}=\mathrm{O}$, $2.6 \%$; $-\mathrm{COO}-, 9.6 \%$ ). When further raising the KPS concentration to 0.1 and $0.2 \mathrm{M}$, the amounts of functional groups showed no obvious increment. The results indicate that KPS is still a mild oxidant to MWCNTs and could oxidize MWCNTs to a certain extent, ensuring the modification process is less destructive.

Raman spectroscopy, as for a powerful tool for the characterization of carbon materials [19-22], was applied to provide structural information about MWCNTs before and after hydrothermal treatment. The two characteristic bands, D-band (disorder band) at $1350 \mathrm{~cm}^{-1}$ and G-band (graphite band) at $1580 \mathrm{~cm}^{-1}$, are observed in 
Figure 1c. Normally, the increase of relative intensity of the $D$ and $G$ band $\left(I_{D} / I_{G}\right.$ ratio) has often taken as a fingerprint for successful modification [21,22]. It was obvious that the $\mathrm{I}_{\mathrm{D}} / \mathrm{I}_{\mathrm{G}}$ ratios of MWCNTs increased after hydrothermal modification, which confirmed the successful introduction of functional groups on the MWCNT surface. More interestingly, the $\mathrm{I}_{\mathrm{D}} / \mathrm{I}_{\mathrm{G}}$ ratio did not depend on the KPS concentration, as clearly shown in Figure 1c. Besides the first-order D-band, the spectra of the samples also show another first-order band at about $1620 \mathrm{~cm}^{-1}$, which is called D'band and also accounts for the structural disorders. The D'-band corresponds to a graphitic lattice mode with $E_{2 g}$ symmery [21]. As shown in Figure 1c, the intensity of D'-band showed the same increase tendency as that of D-band, which can be also attributed to the increase of the structural disorders after modification. Thus, the Raman analyses indicated no obvious structural defects were formed on the MWCNT surface even after hydrothermal treatment at high KPS concentration, which agreed well with XPS analysis. These XPS and Raman results indicated that various hydrophilic functional groups were introduced onto the modified MWCNTs without obvious structural defects.

The effect of $\mathrm{KOH}$ concentration and reaction time on MWCNT modification was studied as well. The XPS and Raman results are presented in Table 1. For the effect of $\mathrm{KOH}$ concentration, the results of No.5-7 experiments where the $\mathrm{KOH}$ concentrations were controlled at $0.4,0.6$ and $0.8 \mathrm{M}$ (the molar ratios of $\mathrm{KPS} / \mathrm{KOH}$ were $1: 2,1: 3$ and 1:4). With the increase of $\mathrm{KOH}$ concentration, the functional groups especially the C-O- group increase up to certain content, which is in good agreement with the reported results [23]. Here, we focus our attention on the introduction of functional groups with high ionization degree by $\mathrm{KOH}$. Generally, the final functional groups created on the MWCNT surface clearly correlate with the $\mathrm{pH}$ of reaction system [14]. In order to get functional groups with high ionization degree (e.g. the ionization degree of $-\mathrm{COOK}$ is much bigger than $-\mathrm{COOH}$ functional group; $-\mathrm{COOH}$ groups formed at acid condition, while - COOK formed at alkaline condition), we should ensure that the MWCNT modification is conducted at the alkaline condition. According the change of $\mathrm{pH}$ value before and after reaction (Nos.5-7 in Table 1) and the mechanism of KPS oxidation [24-26], it is suitable to control the KPS/KOH molar ratio at 1:3. 
Considering the effect of reaction time, it was no need to prolong the time over 0.5 $\mathrm{h}$ according to the XPS and Raman results. The main reason is that the decomposition of KPS could be accomplished within $0.5 \mathrm{~h}$ in hydrothermal process at $160^{\circ} \mathrm{C}$ [24].

\subsection{Detailed characterization of modified MWCNTs}

Taking No.8 modified MWCNTs (m8-MWCNTs) as an example; we examined the dispersion properties of modified MWCNTs in many kinds of solvents as shown in Figure 2a. Unlike pristine MWCNTs, m8-MWCNTs show high solubility in polar solvents, such as water, ethanol, dimethylformamide (DMF), tetrahydrofuran (THF), dimethylacetamide (DMAc). The m8-MWCNTs show the highest solubility in water and the solubility is around $1.16 \mathrm{mg} / \mathrm{ml}$, measured by the reported methods [27]. However, m8-MWCNTs cannot be well dispersed in nonpolar solvents (such as hexane and toluene) and eventually precipitated. This indicated that various hydrophilic groups were created on the m8-MWCNTs after hydrothermal modification. For further understanding of the dispersion property in water, we measured the $\zeta$-potential of m8-MWCNTs in aqueous solution with various $\mathrm{pH}$ and temperature. The $\zeta$-potential provides an indicator of stability of colloidal systems. Greater magnitude (the absolute value $\geq 30 \mathrm{mV}$ ) of the $\zeta$-potential endows the colloid system with an improved stability against coagulation. Measurements of the $\zeta$ potential have already been used to discuss the stability of MWCNT/water system [28-30]. The $\zeta$-potential of aqueous m8-MWCNT solution as a function of $\mathrm{pH}$ is shown in Figure $2 \mathrm{~b}$. At $\mathrm{pH} \geq 4$, the absolute value of the $\zeta$-potential of m8-MWCNTs is larger than $30 \mathrm{mV}$, which indicates that m8-MWCNT aqueous solution very stable. From the photograph of the solution at $\mathrm{pH}$ of 4 , the solution form stable suspensions without visible aggregation even after two weeks (Figure $2 \mathrm{~b}$ inset). On the other hand, when $\mathrm{pH} \leq 3.5$, the $\mathrm{MWCNT} /$ water system turns to be unstable and finally precipitated (Figure $2 \mathrm{~b}$ inset). The aqueous dissolution mechanism of m8-MWCNTs in water could be attributed to the dominance of steric and electrostatic repulsion between the modified MWCNTs over the van der Waals attraction that leads to coagulation. Therefore, the drastic change of the stability of m8-MWCNTs aqueous solution at $\mathrm{pH} \leq 3.5$ could be attributed to the decrease of electrostatic repulsion, which results from the less charge of the functional groups at low $\mathrm{pH}$, such as COOK are converted into $-\mathrm{COOH}$ functional groups. The $\zeta$-potential of aqueous m8- 
MWCNT solution as a function of temperature is shown in Figure 2c. The m8MWCNT solution was very stable below $50{ }^{\circ} \mathrm{C}$, however, it tend to re-aggregate above $60{ }^{\circ} \mathrm{C}$. This may be resulted from the less ionization of $-\mathrm{COOK}$ groups due to the acceleration of hydrolysis reaction at high temperature.

Direct morphologies of pristine MWCNTs and m8-MWCNTs were observed by SEM (Figure 3a,b) and TEM (Figure 3c,d). Figure 3a reveals that the pristine MWCNTs are very hard to disperse uniformly, tend to aggregate together. In contrast, m8-MWCNTs (Figure 3b) are homogeneous dispersed due to the hydrophilic functional groups. Comparison of TEM images in Figure $3 \mathrm{c}$ and Figure $3 \mathrm{~d}$ indicates that the length and diameter of MWCNTs were well retained in m8-MWCNTs. HRTEM images (Figure 3c,d insets) reveal that both morphology and crystal structure of the MWCNT wall were well reserved in modified MWCNTs. This character is quite unusual since other oxidative modification methods usually lead to a significant decrease in tube length and/or destroy of crystal structure of MWCNT walls. In this sense, the present method utilizing alkaline-mediated hydrothermal condition is superior to the other oxidative modification methods.

We conducted thermogravimetrical analysis (TGA) in air and nitrogen condition to measure the oxidation resistance as well as the quantity of functional groups created on the nanotube surface. TGA under air flow shows that the degradation temperature of m8-MWCNTs is much lower than the pristine nanotube (Figure 4a). We calculated the temperature at maximal weight loss rate $\left(T_{\text {mwlr }}\right)$ from their corresponding differential thermogravimetric (DTG) curves and found that $T_{\text {mwlr }}$ value decreased from $680{ }^{\circ} \mathrm{C}$ to $450{ }^{\circ} \mathrm{C}$ after hydrothermal modification. Although the mechanism of the sharp decrease of $T_{\text {mwlr }}$ value is still under investigation, it seems to be attributed to the functional groups, because the functional groups and/or defective site become very reactive to oxygen at elevated temperature [31]. TGA curves conducted under nitrogen atmosphere are presented in Figure $4 \mathrm{~b}$. According to the DTG curves of pristine MWCNTs and m8-MWCNTs, we believe that the mass loss over $610{ }^{\circ} \mathrm{C}$ should be assigned to the decomposition of MWCNT structure. Thus, the quantity of functional groups on the m8-MWCNT surface was estimated to be nearly $11.4 \mathrm{wt} \%$.

\section{Conclusions}


In summary, we have reported a simple and effective hydrothermal process to modified MWCNTS. The modified MWCNTs are highly soluble in polar solvents, such as water, ethanol, dimethylformamide. XPS, Raman, SEM and TEM results confirm that the functional groups were successfully created onto the MWCNT surface without obvious structure damage. A series of modification experiment were carefully investigated to determine the rational reaction parameters. This convenient approach could be easy to enlarge from milligram scale to gram level, expected to be a practical and common approach to modify carbon nanotubes in the future. 


\section{References}

[1] M.T. Byrne, Y.K. Gun'ko, Adv. Mater. 22 (2010) 1672.

[2] X. Xu, A.J. Uddin, K. Aoki, Y. Gotoh, T. Saito, M. Yumura, Carbon 48 (2010) 1977.

[3] W. Lu, N. Li, W. Chen, Y. Yao, Carbon 47 (2009) 3337.

[4] L. Zhang, Q.Q. Ni, A. Shiga, Y. Fu, T. Natsuki, Polym. Compos. 31 (2010) 491.

[5] A.E. Aliev, M.D. Lima, S. Fang, R.H. Baughman, Nano Lett. 10 (2010) 2374.

[6] Y. Asada, Y. Miyata, Y. Ohno, R. Kitaura, T. Sugai, T. Mizutani, H. Shinohara, Adv. Mater. 22 (2010) 2698.

[7] S.H. Lee, K. Teshima, I.Y. Jang, K. Yubuta, Y.J. Kim, Y.A. Kim, T. Shishido, M. Endo, S. Oishia, Chem. Commun. 46 (2010) 2295.

[8] L. Matlhoko, S.K. Pillai, M. Moodley, W.G. Augustyn, S.S.Ray, J. Nanosci. Nanotechno. 9 (2009) 5431.

[9] Y. Lian, Y. maeda, T. Wakahara, T. Akasaka, S. Kazaoui, N. Minami, T. Shimizu, N. Choj, H. Tokumoto, J. Phys. Chem. B 108 (2004) 8848.

[10] B. Jia, L. Gao, J. Phys. Chem. B 111 (2007) 5337.

[11] M.N. Tchoul, W.T. Ford, G. lolli, D.E. Resasco, S. Arepalli, Chem. Mater. 19 (2007) 5765 .

[12] A.G. Osorio, I.C.L. Silveira, V.L. Bueno, C.P. Bergmann, Appl. Surf. Sci. 255 (2008) 2485.

[13] J. Zhang, H. Zou, Q. Qing, Y. Yang, Q. Li, Z. Liu, X. Guo, Z. Du, J. Phys. Chem. B 107 (2003) 3712.

[14] L. Zhang, Q.Q. Ni, Y. Fu, T. Natsuki, Appl. Surf. Sci. 255 (2009) 7095.

[15] C. Chen, B. Liang, D. Lu, A. Ogino, X. Wang, M. Nagatsu, Carbon 48 (2010) 939.

[16] H. Liu, X. Wang, P. Fang, S. Wang, X. Qi, C. Pan, G. Xie, K.M. Liew, Carbon 48 (2010) 721.

[17] L. Minati, S. Torrengo, G. Speranza, Surf. Sci. 604 (2010) 508.

[18] K.A. Wepasnick, B.A. Smith, J.L. Bitter, D.H. Fairbrother, Anal. Bioanal. Chem. $396(2010) 1003$.

[19] Md. M.R. Khan, Y. Gotoh, H. Morikawa, M. Miura, Y. Fujimori, M. Nagura, Carbon 45 (2007) 1035.

[20] Y. Liu, W. Wongwiriyapan, K.C. Park, H. Muramatsu, K. Takeuchi, Y.A. Kim, M. Endo, Carbon 47 (2009) 2528. 
[21] H.M. Heise, R. Kuckuk, A.K. Ojha, A. Srivastava, V. Srivastavad, B.P. Asthanac, J. Raman Spectrosc. 40 (2009) 344.

[22] M.S. Dresselhaus, G. Dresselhaus, R. Saito, A. Joriod, phys. Rep. 409 (2005) 47.

[23] D. Yang, G. Guo, J. Hu, C. Wang, D. Jiang, J. Mater. Chem. 18 (2008) 350.

[24] J. Kronholm, M.L. Riekkola, Environ. Sci. Technol. 33 (1999) 2095.

[25] D.D. Tanner, S.A.A. Osman, J. Org. Chem. 52 (1987) 4689.

[26] P. Yang, J.Y. Deng, W.T. Yang, Polymer 44 (2003) 7157.

[27] J. Zou, L. Liu, H. Chen, S.I. Khondaker, R.D. McCullough, Q. Huo, L. Zhai, Adv. Mater. 20 (2008) 2055.

[28] K.C. Etika, M.A. Cox, J.C. Grunlan, Polymer 51 (2010) 1761.

[29] M.U. Khan, V.G. Gomes, I.S. Altarawneh, Carbon 48 (2010) 2915.

[30] H. Orikasa, N. Inokuma, S. Okubo, O. Kitakami, T. Kyotani, Chem. Mater. 18 (2006) 1036.

[31] D. Yang, G. Guo, J. Hu, C. Wang, D. Jiang, J. Mater. Chem. 18 (2008) 350. 


\section{Figure Captions}

Figure 1. XPS and Raman spectra of No.1-4 samples in Table 1: (a) survey scan, (b) C 1s narrow scan and (c) Raman spectra.

Figure 2. (a) Photograph of the dispersed m8-MWCNTs in five solvents after 2 weeks; values of $\zeta$-potential for the m8-MWCNTs aqueous solution: (b) at different $\mathrm{pH}$ and (c) at different temperature.

Figure 3. Typical FESEM images of (a) pristine MWCNTs and (b) m8-MWCNTs, with their high magnification images in the inset; typical TEM images of (c) pristine MWCNTs and (d) m8-MWCNTs, with their individual MWCNT images in the inset.

Figure 4. TGA and DTG curves of pristine MWCNTs and m8-MWCNTs: (a) under air flow and (b) under $\mathrm{N}_{2}$ flow. 
Table 1. Detailed experimental conditions and corresponding analyses.

\begin{tabular}{|c|c|c|c|c|c|c|c|c|c|c|c|}
\hline \multirow[b]{2}{*}{ No. } & \multicolumn{2}{|c|}{ Reagents } & \multirow[b]{2}{*}{ Initial pH } & \multirow[b]{2}{*}{ Final $\mathrm{pH}$} & \multirow{2}{*}{$\begin{array}{c}\text { Reaction } \\
\text { Time } \\
\text { (h) }\end{array}$} & \multicolumn{5}{|c|}{ Relative percentages of functional groups on MWCNT surface by XPS (\%) } & \multirow{2}{*}{$\begin{array}{l}\text { ID/IG Ratio } \\
\text { by Raman }\end{array}$} \\
\hline & $\begin{array}{l}\text { KSP Conc. } \\
\text { (M) }\end{array}$ & $\begin{array}{c}\text { KOH Conc. } \\
\text { (M) }\end{array}$ & & & & $\mathrm{C}-\mathrm{C} \mathrm{sp}^{2}$ & $\mathrm{C}-\mathrm{C} \mathrm{sp}^{3}$ & $\mathrm{C}-\mathrm{O}$ & $\mathrm{C}=\mathrm{O}$ & $\mathrm{O}-\mathrm{C}=\mathrm{O}$ & \\
\hline $0^{a}$ & ----- & ----- & ----- & ----- & ----- & 57.7 & 30.4 & 4.9 & 2.7 & 4.3 & 0.90 \\
\hline 1 & 0.01 & 0.03 & 12.5 & 7.4 & 4 & 51.3 & 32.4 & 6.4 & 2.0 & 7.9 & 1.10 \\
\hline 2 & 0.05 & 0.15 & 13.2 & 11.3 & 4 & 49.4 & 30.8 & 7.6 & 2.6 & 9.6 & 1.23 \\
\hline 3 & 0.1 & 0.3 & 13.5 & 12.3 & 4 & 47.5 & 32.4 & 7.7 & 2.7 & 9.6 & 1.14 \\
\hline 4 & 0.2 & 0.6 & 13.8 & 13.0 & 4 & 46.0 & 34.1 & 7.7 & 2.8 & 9.3 & 1.13 \\
\hline 5 & 0.2 & 0.4 & 13.6 & 2.0 & 4 & 42.3 & 38.7 & 6.7 & 3.1 & 8.9 & 1.21 \\
\hline 6 & 0.2 & 0.6 & 13.8 & 13.0 & 4 & 46.0 & 34.1 & 7.7 & 2.8 & 9.3 & 1.13 \\
\hline 7 & 0.2 & 0.8 & 13.9 & 13.6 & 4 & 44.6 & 33.6 & 8.8 & 2.4 & 10.4 & 1.16 \\
\hline 8 & 0.05 & 0.15 & 13.2 & 11.5 & 0.5 & 45.2 & 33.9 & 8.3 & 2.8 & 9.8 & 1.15 \\
\hline 9 & 0.05 & 0.15 & 13.2 & 11.5 & 1 & 46.0 & 33.7 & 7.8 & 2.8 & 9.7 & 1.17 \\
\hline 10 & 0.05 & 0.15 & 13.2 & 11.6 & 2 & 46.9 & 33.1 & 7.3 & 2.8 & 9.7 & 1.14 \\
\hline 11 & 0.05 & 0.15 & 13.2 & 11.3 & 4 & 49.4 & 30.8 & 7.6 & 2.6 & 9.6 & 1.23 \\
\hline
\end{tabular}

Note: No. 1-4, No. 5-7 and No. 8-11 experiments with different KPS concentration, KOH concentration and reaction time, respectively.

${ }^{\text {a }}$ No.0 represents pristine CNT 

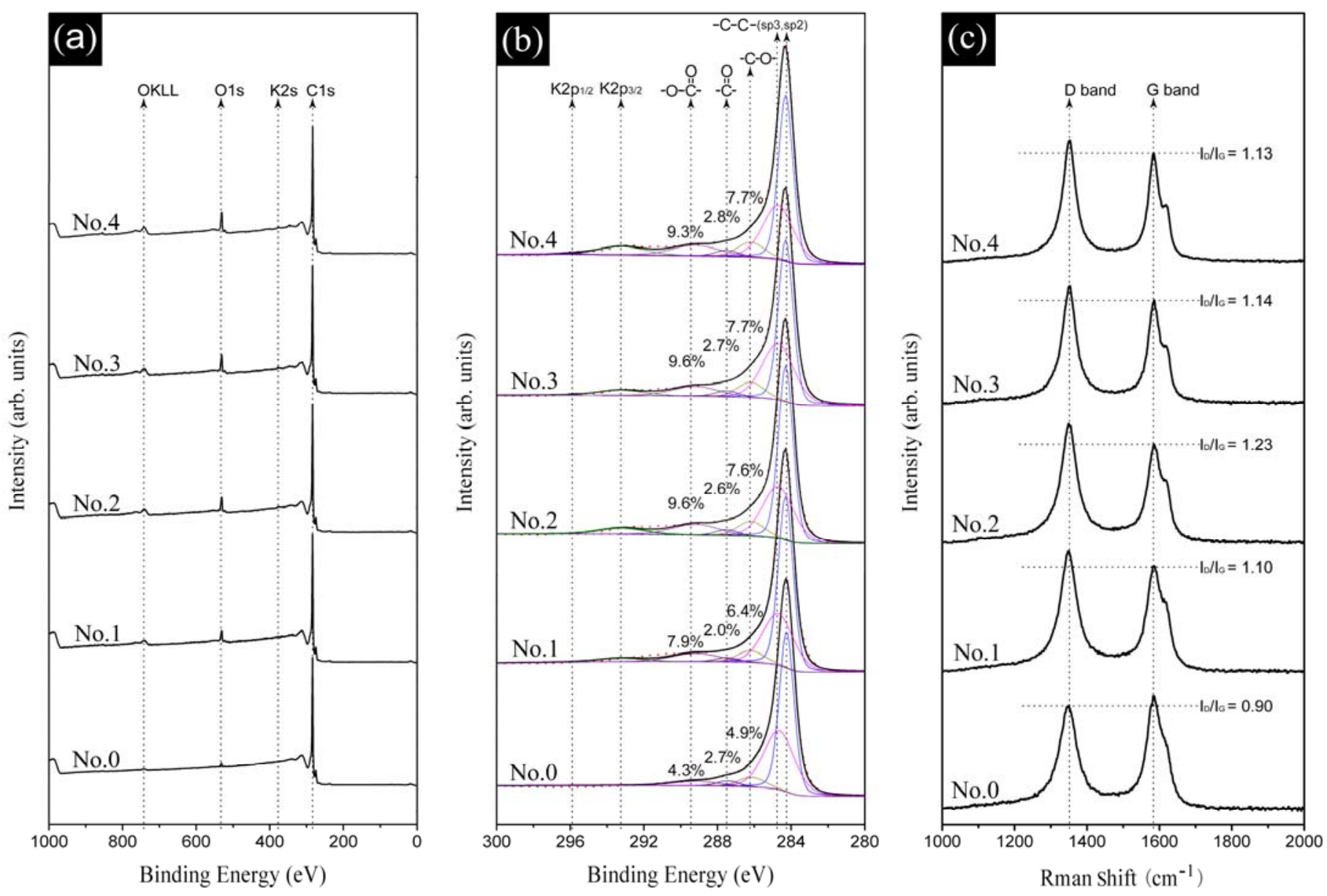

Figure 1. 

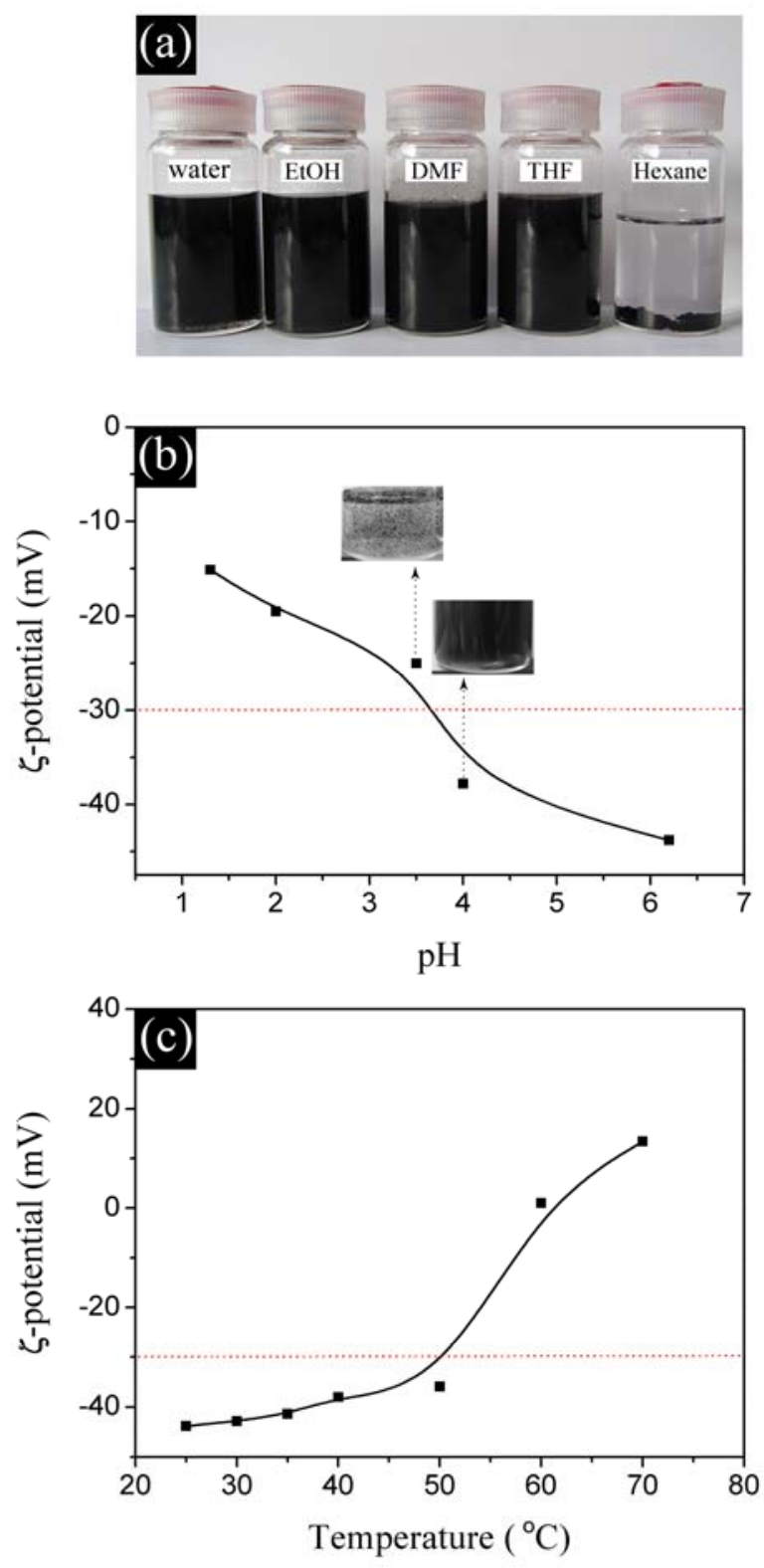

Figure 2. 

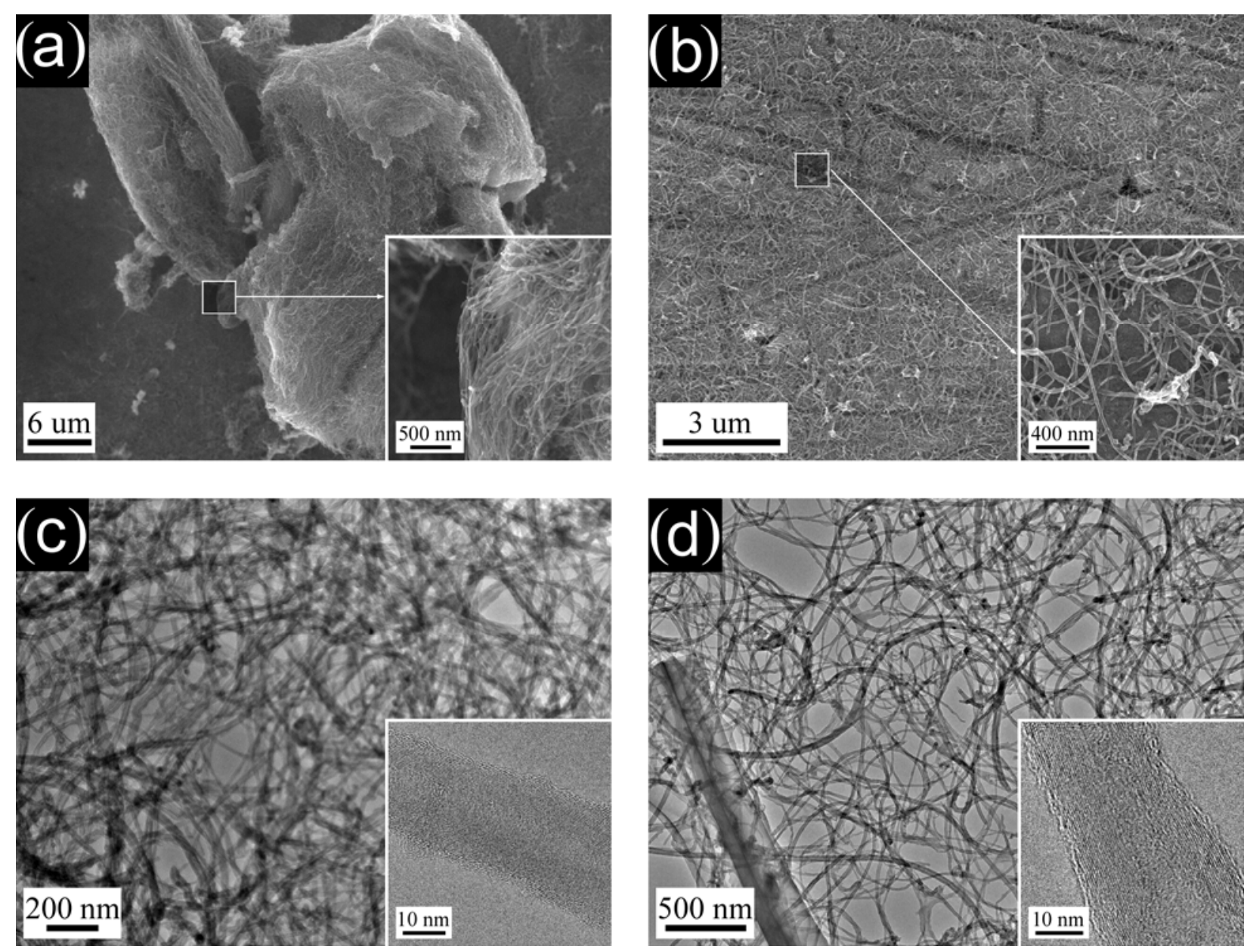

Figure 3. 

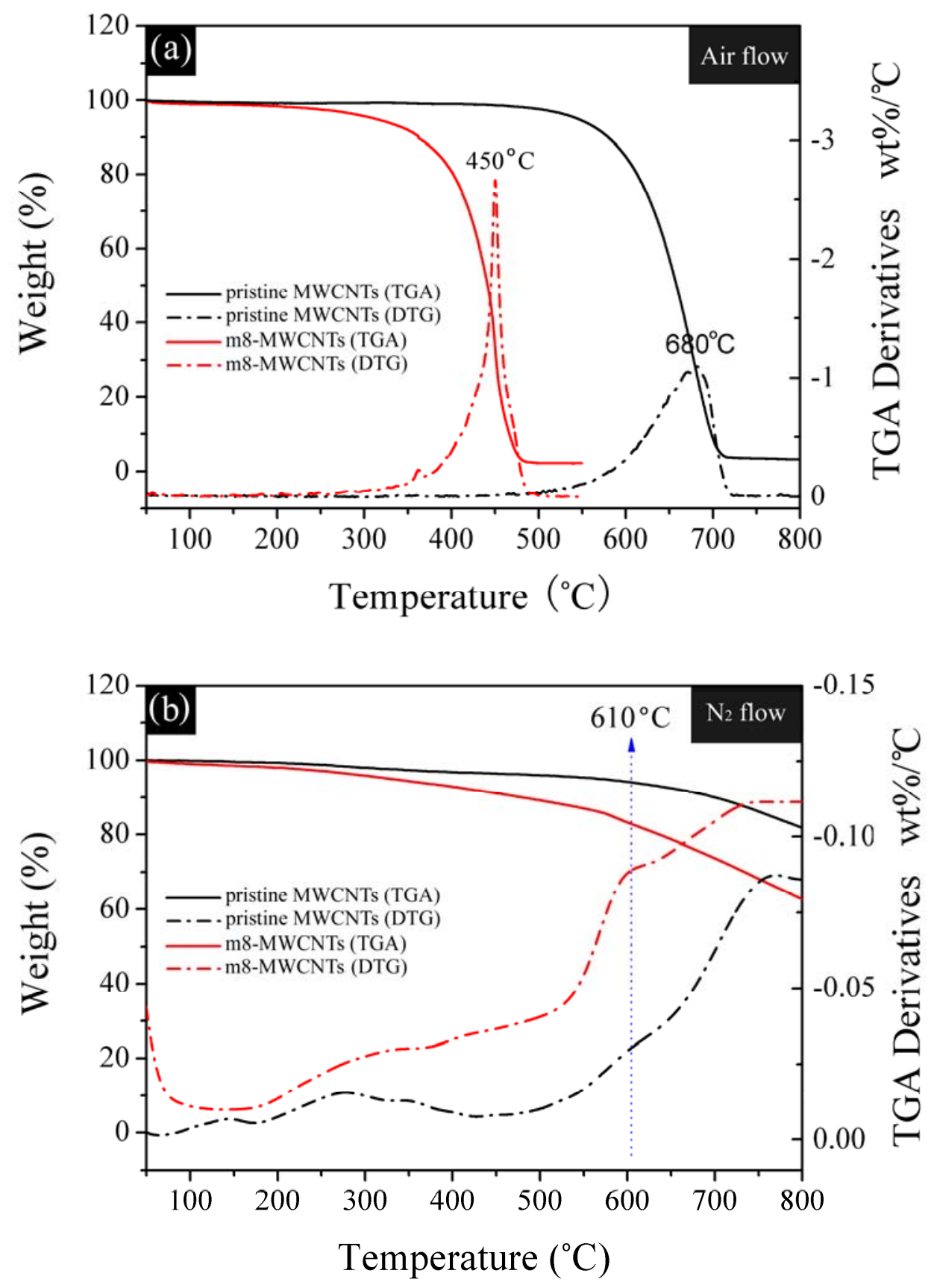

Figure 4. 